\title{
论文
}

\section{西藏芒康似勾儿茶叶属(鼠李科)化石及其 生物地理学意义}

周浙昆 ${ }^{1,2^{*} \dagger}$, 王腾翔 ${ }^{1,3 \dagger}$, 黄健 ${ }^{1}$, 刘佳 ${ }^{1}$, 邓炜显东 ${ }^{1,3}$, 李仕虎 ${ }^{4,5}$, 邓成龙 ${ }^{4}$, 苏涛 1,38

1. 中国科学院西双版纳热带植物园热带森林生态学重点实验室, 西双版纳 666303;

2. 中国科学院昆明植物研究所东亚植物多样性与植物地理重点实验室, 昆明 650223;

3. 中国科学院大学, 北京 100049 ;

4. 中国科学院地质与地球物理研究所岩石圈演化国家重点实验室, 北京 100029 ;

5. Lancaster Environment Centre, Lancaster University, Lancaster LA1 4YQ, UK

†共同第一作者

* 通讯作者, E-mail: zhouzk@xtbg.ac.cn

$\S$ 通讯作者, E-mail: sutao@xtbg.org.cn

收稿日期：2019-05-07; 收修改稿日期：2019-08-16; 接受日期：2019-08-23; 网络版发表日期：2019-11-26

中国科学院战略性先导科技专项项目(编号：XDA2007030102、XDB26000000)、国家自然科学基金-英国自然环境研究理事会国际合作重点 项目(编号：41661134049、NE/P013805/1)、中国科学院第二次青藏高原综合科学考察研究项目(STEP)(编号：2019QZKK0705)、中国科学院 青年创新促进会项目(编号: 2017439)和中国科学院前沿科学重点研究计划项目(编号: QYZDB-SSW-SMC016)资助

摘要文章报道了发现于西藏东南部芒康盆地上始新统的鼠李科植物叶化石标本. 化石叶缘全缘或微波状, 二级 脉间距规则, 弧曲向上并与其上的二级脉在叶缘处结合并与叶缘融合, 形成真曲脉序; 二级脉在中上部较为密集, 三级脉密集相互平行, 横贯二级脉之间, 这些叶脉特征与美洲分布的灭绝类群——似勾儿茶叶属(Berhamniphyllum Jones and Dilcher)非常相似. 百分之四十的二级脉集中于叶的上半部是当前化石的一个典型特征，未见于该 属的其他类群, 于是将当前化石定为君容似勾儿茶叶新种(Berhamniphyllum junrongiae Z. K. Zhou, T. X. Wang et J. Huang sp. nov.). 进一步研究发现, 仅凭叶脉特征不能将勾儿茶属(Berchemia)和Rhamnidium、Karwinskia等三个属 区分开来, 似勾儿茶叶属(Berhamniphyllum)代表了这几个属的一个灭绝的共同祖先, 本文对产于云南和山东等地 三种勾儿茶属化石进行了归并, 并将其归并入似勾儿茶叶属中. 根据形态学、分子系统证据和化石记录, 将勾儿 茶属、Rhamnidium、Karwinskia和似勾儿茶叶属定义为勾儿茶复合群(Berchemia complex). 本文还讨论了勾儿茶 复合群地理分布格局的演变历史, 认为勾儿茶复合群于晚白严世晚期起源于南美哥伦比亚, 在始新世经中美洲扩 散到北美, 后又从北美经过北大西洋陆桥扩散至欧洲并从欧洲扩散至非洲。东亚的勾儿茶复合群是经白令陆桥扩 散而来, 时间不晚于始新世, 这一类群在东亚最早出现于西藏芒康, 其后再扩散至亚洲其他地区.

关键词勾儿茶复合群, 似勾儿茶叶属, 新生代, 青藏高原, 生物地理 


\section{1 引言}

一个类群的起源、演化以及现代分布格局的形成 与其地质历史分布有着不可分割的联系. 因此,化石在 生物地理的研究中起着至关重要的作用(Taylor等, 2008). 对一个类群化石历史的追溯，能够窥豹类群分 布区形成演变的一斑. 对类群地质历史的研究, 已经成 为生物地理学研究的重要方法和手段.

青藏高原的隆升改变了亚洲的地形地貌和大气环 流, 对亚洲生物多样性的格局产生了重要的影响. 青藏 高原隆升及其环境效应对生物多样性影响的研究一直 是地球科学和生命科学研究的热点. 近年来, 不少新的 化石类群在青藏高原被发现和报道，这些古生物学的 新进展不仅改变了人们对青藏高原地形地貌的认识, 而且还为青藏高原生物多样性格局的演变提供了新视 野(Ding等, 2017; Spicer, 2017; Wu等, 2017; Ai等, 2019; Deng等, 2019; Jia等, 2019; Jiang等, 2019; Liu等, 2019; Su等, 2019a, 2019b; Tang等, 2019; Xu C L等, 2019; Xu $\mathrm{H}$ 等, 2019). 最新的研究表明, 青藏高原与北半球其他 地区的植物区系在古近纪就已经建立了紧密的联系， 古近纪青藏高原是北半球植物区系交流的一个重要的 区系港(Floristic Hub). 有一些类群从印度板块进入青 藏高原, 如臭椿属(Ailanthus)(Liu等, 2019); 而一些类群
从北美扩散到青藏高原，再从青藏高原扩散到东亚其 他地区，如椿榆属(Cedrelospermum)(Jia等，2015, 2019); 还有一些类群始新世在北美、欧洲和青藏高原 都有发现，但是现在已经灭绝，如兔耳果属(Lagokarpos)(Tang等，2019). 然而，和青藏高原广衰的地域相 比, 其新生代植物研究仍是较为薄弱的, 加强青藏高原 新生代植物化石的研究, 方能更加深入地了解青藏高 原与北半球其他地区植物区系的交流与联系.

勾儿茶属 (Berchemia) 是鼠李科鼠李族(Rhamneae Hook f.)下的一个小属. 大多数勾儿茶属植物为木质藤 本, 少数为小灌木或小乔木(Chen和Schirarend, 2007). 全属约32种，主要分布于东亚的热带到温带地区以及 东南亚的热带地区，其中1种(Berchemia scandens)分 布于美国东南部、墨西哥和危地马拉(Flora of North America Editorial Committee, 1993+), 另2种分布于南 非(Palgrave, 2015). 吴征镒等(2006)虽然将其分布类型 划归为 9 型即东亚-北美间断分布型，但是明确记载非 洲也有勾儿茶属植物的分布. 该属植物的分布区类型 与热带亚洲-热带非洲-热带美洲(2-2)的分布区类型更 为相近(图1). 在Richardson等(2000)分子系统学中, 勾 儿茶属被从杳族中移到鼠李族中. 鼠李族主要分布于 泛热带和北半球的温带地区，其中勾儿茶属 (Berchemia)、小勾儿茶属(Berchemiella)、猫乳属(Rhamnel-

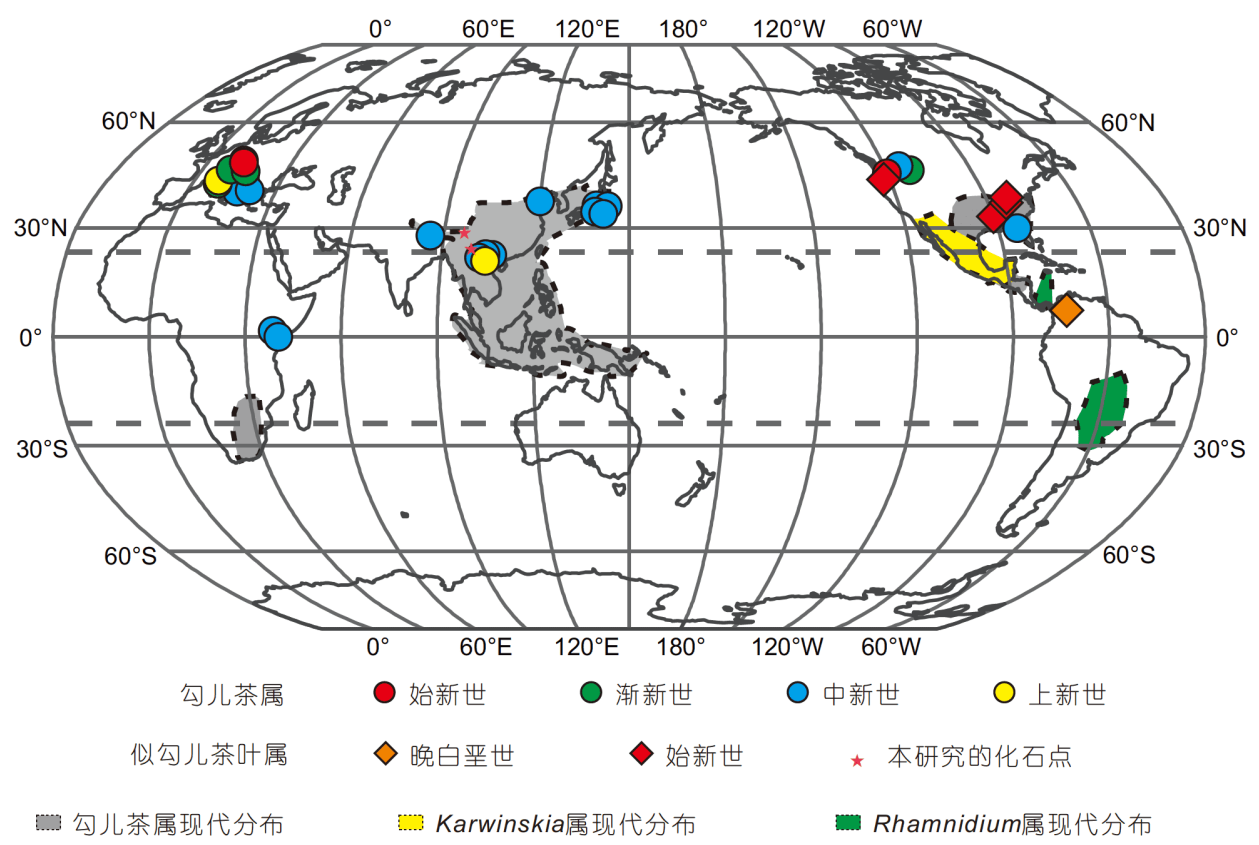

图 1 勾儿茶属及其相似类群的化石记录和现代分布 
$l a)$ 、鼠李属 $($ Rhamnus $)$ 、雀梅藤属(Sageretia) 和对刺藤 属(Scutia) 等6个属在中国有分布.

勾儿茶属及与其有密切亲缘关系的类群有着广泛 的化石记录(图1; 表1和2). 最早的与勾儿茶属有密切 亲缘关系的化石记录可以追溯到哥伦比亚的晚白严世 最晚期, 这是一个被定为似勾儿茶叶未定种的叶化石 (Correa等，2010)．德国中始新世Messel植物群所产的 内果皮(endocarp)化石是勾儿茶属植物最早的化石记 录(Collinson等, 2012). 勾儿茶属及其近缘类群的化石 在北美、欧洲、亚洲和非洲新生代地层中都有报道
(Becker，1969; Manchester，2000; Bozukov等，2008; Collinson等, 2009; Lazarević等, 2013; 中国新生代植物 编写组, 1978)(图1, 表1和2). 似勾儿茶叶未定种(Berhamniphyllum sp.) 是鼠李族最早的化石，而且可能是 Rhamnidium 和勾儿茶属的共同祖先. 在现代植物中, 这两个类群在形态特征上也极为相似, 在分子系统学 上又都位于亲缘关系密切的同一个单系的分枝内. 勾 儿茶属的类群大部分分布于东亚和东南亚, 个别类群 分布于北美，极个别种类分布于南非. 勾儿茶属化石 历史反映的现代分布格局的形成过程和金虎尾科植物

表 1 勾儿茶属化石记录

\begin{tabular}{|c|c|c|c|c|}
\hline 物种 & 器官 & 时代 & 地点 & 文献 \\
\hline Berchemia mellerae & 内果皮 & 中始新世 & 德国Messel & Collinson等, 2012 \\
\hline B. sp & 叶 & 晚始新世 & 美国俄勒冈州 & Manchester, 2000 \\
\hline B. altorhenana & 叶 & 早渐新世 & 德国Rauenberg & Kovar-Eder, 2016 \\
\hline B. multinervis & 叶 & 晚渐新世 & 瑞士Ebnat-Kappel & Büchler, 1990 \\
\hline B. multinervis & 叶 & 晚渐新世 & 保加利亚Vulche Pole Molasse组 & Bozukov等, 2008 \\
\hline B. multinervis & 叶 & 晚渐新世 & 罗马尼亚Petrosani盆地 & Givulescu, 1996 \\
\hline B. huanoides & 叶 & 晚渐新世 & 美国蒙大拿州 & Becker, 1969 \\
\hline B. multinervis & 叶 & 中新世 & 保加利亚Western Rhodopes & Bozukov, 2000 \\
\hline B. priscaformis & 叶 & 中新世 & 美国南卡罗莱纳州 & Berry, 1916b \\
\hline B. sp & 叶 & 中新世 & 美国爱达荷州 & Smiley等, 1975 \\
\hline B. pseudodiscolor & 种子 & 早中新世 & 肯尼亚Rusinga岛 & Collinson等, 2009 \\
\hline B. acutangula & 叶 & 早中新世 & 巴伐利亚Goldern & Spitzelberger, 1989 \\
\hline B. miofloribunda & 叶 & 早中新世 & 日本本州 & Yabe, 2008 \\
\hline B. multinervis & 叶 & 早中新世 & 塞尔维亚Valjevo-Mionica盆地 & Lazarević等, 2013 \\
\hline B. multinervis & 叶 & 早中新世 & 捷克Most盆地 & Sakala, 2000; Teodoridis, 2007 \\
\hline B. multinervis & 叶 & 早-中中新世 & 瑞士Canton Lucerne & Köecke和Uhl, 2015 \\
\hline B. pseudodiscolor & 外果皮 & 中中新世 & 肯尼亚Fort Ternan & Retallack, 1992 \\
\hline B. miofloribunda & 叶 & 中中新世 & 日本能登半岛 & Ishida, 1970 \\
\hline B. nepalensis & 叶 & 中中新世 & 尼泊尔Koilabas & Prasad和Dwivedi, 2007 \\
\hline B. multinervis & 叶 & 晚中新世 & 巴伐利亚Lerch & Jung, 1968 \\
\hline B. multinervis & 叶 & 晚中新世 & 瑞士Oehingen & Hantke, 1954; Heer, 1855-1859 \\
\hline B. miofloribunda & 叶 & 晚中新世 & 日本本州 & Ozaki, 1980 \\
\hline B. miofloribunda & 叶 & 晚中新世 & 日本本州 & Ozaki, 1991 \\
\hline B. miofloribunda & 叶 & 上新世 & 中国云南团田 & 吴靖宇, 2009 \\
\hline B. cf. yunnanensis & 叶 & 上新世 & 中国云南团田 & 吴靖宇, 2009 \\
\hline B. multinervis & 叶 & 上新世 & 意大利Fossano & Macaluso等, 2018 \\
\hline B. floribunda & 叶 & 晚第三纪 & 印度Jharkhand & Singh和Prasad, 2007 \\
\hline
\end{tabular}


表 2 其他勾儿茶类化石记录

\begin{tabular}{cccc}
\hline 物种 & 时代 & 地点 & 文献 \\
\hline Berhamniphyllum sp. & 晚白普世 & 南美哥伦比亚 & Correa等, 2010 \\
Berhamniphyllum claibornense & 早始新世 & 美国肯塔基州、田纳西州 & Berry, 1916a; Jones和Dilcher, 1980 \\
Berhamniphyllum claibornense & 中始新世 & Dilcher和Lott, 2005 \\
Berhamniphyllum sp. & 中始新世 & 美国田纳西州 & Dilcher和Lott, 2005 \\
Berhamniphyllum sp. & 晚始新世 & 美国田纳西州 & Myers等, 2002 \\
Berhamniphyllum junrongiae sp. & 晚始新世 & 美国俄勒冈州 & 本研究 \\
nov. & 中国西藏芒康 & de León等, 1998 \\
Karwinskia axamilpense & 渐新世 & 墨西哥普埃布拉 & 中国新生代植物编写组, 1978; 周浙昆, 1985; \\
Berhamniphyllum miofloribundum \\
comb. nov.
\end{tabular}

非常相似(Davis等, 2002; Zhou等, 2006), 两者都是通 过金虎尾路线即起源于南美洲北部，然后扩散到北美， 从北美通过北大西洋路桥扩散到欧洲和热带亚洲，其 中部分类群从欧洲向南扩散至非洲, 形成今天的分布 格局(Zhou等, 2006). 研究这个类群的化石历史, 探讨 他们的亲缘关系, 讨论其地理分布格局的形成过程, 就 成了一个饶有兴趣的植物地理学问题. 对这一问题的 研究不仅对于理解鼠李族乃至鼠李科的现代分布格局 有着重要意义，而且对于深入了解泛热带间断分布格 局的形成和演变也有重要的参考价值.

芒康盆地位于青藏高原的东南缘, 在构造位置上 属于㒸塘地块. 新生代印度板块和欧亚板块的碰撞在 高原内部和周边地区造成了强烈的构造变形，芒康盆 地的形成与此密切相关. 青藏高原东南缘横断山腹地 是全球生物多样性研究的热点地区(Myers等, 2000), 探讨这一地区地质历史时期植物多样性的演化过程, 不仅对理解生物区系的演变有着重要意义, 而且对重 建高原东南缘古高程和地形地貌乃至认识整个青藏高 原的隆升历史都有着极为重要的意义( $\mathrm{Su}$ 等, $2019 b)$. 最近，笔者在芒康盆地上始新统泥岩中发现了似勾儿 茶叶属化石, 这是该属在亚洲最早的化石记录. 对这 一化石的研究, 能更进一步理解青藏高原植物区系与 北半球其他地区植物区系在古近纪的联系.

本文描述报道这一新发现的化石，并对产自云南 和山东中新世的勾儿茶属的化石种类进行厘定, 讨论 Rhamnidium属、勾儿茶属和Berhamniphyllum 属的分 类关系, 以这一发现为例, 讨论青藏高原植物区系在古 近纪与北半球, 乃至中美洲植物区系的联系, 以及这些 类群地理分布格局的演变.

\section{2 材料与方法}

\section{1 化石产地}

本研究的化石材料分别来自西藏芒康卡均村拉屋 拉组, 云南文山花枝格组以及云南开远小龙潭组(图2).

编号为MK3-1491和MK3-2155的化石产自于西藏 芒康县卡均村拉屋拉组褐黄色泥岩中, Su等(2019b)将 该化石层位标注为MK3. 拉屋拉组主要由河湖相灰白 色和灰黄色砂岩、粉砂岩和泥岩组成, 并夹有大量火 山灰, 其中粉砂岩和泥岩中产大量植物化石, 详细化 石剖面地层岩性见Su等(2014，2018，2019b). MK3化 石层夹在两层火山凝灰岩之间, 通过 ${ }^{40} \mathrm{Ar}{ }^{39} \mathrm{Ar}$ 测年分 别得到下部凝灰岩年代为约 $35.5 \mathrm{Ma}$, 上部年代为约 $34.6 \mathrm{Ma}(\mathrm{Su}$ 等, 2019b), 因此MK3化石层年代为晚始新 世最晚期.

编号为DMS-1713和DMS-3872的化石产自云南文

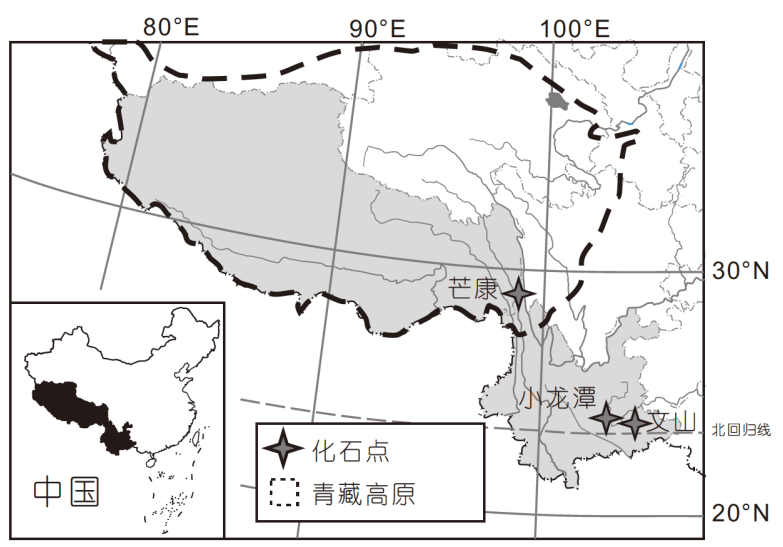

图 2 化石采集点位置 
山大石洞村花枝格组. 花枝格组主要由青灰色、灰白 色或褐黄色湖相泥岩、钙质泥岩与粉砂岩层组成( $\mathrm{Li}$ S F等, 2015; Lebreton-Anberrée等, 2016), 磁性地层学 研究结果表明化石层位年代在15.2 16.5 Ma, 为中中新 世(Lebreton-Anberrée等, 2016).

编号为PB12521的化石产自云南开远小龙潭组. 小龙潭组主要由灰色泥灰岩和黑色褐煤层组成, 古地 磁研究结果表明小龙潭组年代为 $10 \sim 12.7 \mathrm{Ma}(\mathrm{Li} \mathrm{S} \mathrm{H}$ 等, 2015), 与孢粉 (王伟铭，1996)、哺乳动物化石 (Dong和Qi，2013)等研究结果一致，化石层位年代为 晚中新世.

其余订正的材料来自于公开发表的文献.

\section{2 研究方法}

对采集的化石进行编号拍照，勾儿茶属的相关类 群化石记录和讨论涉及的类群通过已经发表的文献进 行查询, 收录在本文的化石记录笔者均查看了原文献, 进行了可靠性的甄别.

现代对比的材料来自标本馆及网上数据库 JSTOR 及中国数字标本馆 CVH，笔者查阅了中国科学院昆明 植物研究所标本馆(KUN)和英国皇家植物园-邱园 $(K)$ 的鼠李科标本，透明叶材料采自中国科学院西双版纳 热带植物园. 化石描述术语参考Ellis等(2009), 系统描 述参考被子植物种系发生学组第四版(Angiosperm Phylogeny Group IV, APG IV).

\section{3 系统古植物学}

目: 蓄薇目Rosales Bercht. \& J. Presl

科: 鼠李科 Rhamnaceae Juss

属: 似勾儿茶叶属Berhamniphyllum Jones and Dilcher

\section{(1) 君容似勾儿茶叶(新种)}

Berhamniphyllum junrongiae Z. K. Zhou, T.X. Wang et J. Huang sp. nov. (图3a 3c和 $3 g$ )

词源学: junrongiae起自中国古植物学家陶君容的 名, 以铭记她对中国新生代古植物学研究的贡献以及 对芒康化石植物群早期的研究.

模式标本: 主模式(Holotype): MK3-1491(图3a 3b 和3g); 副模式(Paratype): MK3-2155(图3c)
模式标本存储地: 中国科学院西双版纳热带植物 园古生态学研究标本室

模式标本产地: 西藏芒康卡均村

地层: 上始新统拉屋拉组

鉴定特征: 叶变异较大, 叶缘全缘或微波状, 二级 脉脉间距规则, 弧曲向上逐步与其上的二级脉在叶缘 处结合并与叶缘融合形成真曲脉序 (图3b和 $3 \mathrm{~g}$ ); 二级 脉在上半部较为密集, 三级脉密集相互平行, 横贯二 级脉之间, 与中脉形成针角.

描述: 叶矩圆形至长卵形, 长2.8 9.3 cm, 宽 $1.4 \sim 2.9 \mathrm{~cm}$, 长宽比2.0:1 3.2:1. 全缘, 有时浅波状; 叶 尖为针角, 急尖或外凸; 叶基为针角, 外凸; 叶尖和叶基 两侧对称. 未见叶柄. 叶脉羽状; 中脉直; 二级脉真曲, 10 14对，叶片中部以上(叶片纵轴 $50 \%$ 以上) 4 8对，占 二级脉总数的 $40 \sim 57 \%$, 间距规则, 直伸与主脉相连, 夹 角向基部从 $17^{\circ}$ 渐增至 $46^{\circ}$ ，伸至近边缘后陡然向上并 与上一级二级脉于叶缘处结合 (图 $3 \mathrm{~b}$ 和 $3 \mathrm{~g}$ ); 间二级脉 极少或无，与二级脉平行. 二级脉间三级脉贯穿，笔直 对生, 有时二歧, 与中脉夹角为针角, 其角度恒定; 更高 级脉序未见.

\section{(2) 多花似勾儿茶叶(新组合)}

Berhamniphyllum miofloribundum (Hu et Chaney) J. Huang, T. Su et Z. K. Zhou comb. nov. (图3d 3f)

1940 Berchemia miofloribunda Hu et Chaney, 65页, 图版9, 图5; 图版40, 图2,3

1978 Berchemia miofloribunda Hu et Chaney, 140 页, 图1, 2; 图版130, 图3

研究材料: DMS-1713 和DMS-3872(云南文山花枝 格组灰黄色泥岩); PB12521(云南开远小龙潭组泥 灰岩)

补充描述：文山(图3d和3e)、小龙潭(图3f)的标本 归于多花似勾儿茶叶. 叶椭圆形至圆形, 长1.6 2.5 cm, 宽1.1 1.4cm, 长宽比1.5:1 2.1:1. 全缘; 叶尖为钝角, 圆 形或外凸; 叶基为针角, 外凸或急尖; 叶尖和叶基两侧 对称. 未见叶柄. 叶脉羽状; 中脉直; 二级脉真曲, 5 9 对，叶片中部以上 $1 \sim 3$ 对，占二级脉总数的 $20 \sim 33 \%$, 间 距规则, 直伸与主脉相连, 夹角向基部从 $15^{\circ}$ 渐增至 $52^{\circ}$, 伸至近边缘后陡然向上并与上一级二级脉于叶缘处结 合; 间二级脉无.二级脉间三级脉贯穿, 笔直对生, 有时 二歧，与中脉夹角为针角，其角度恒定; 更高级脉序 未见. 

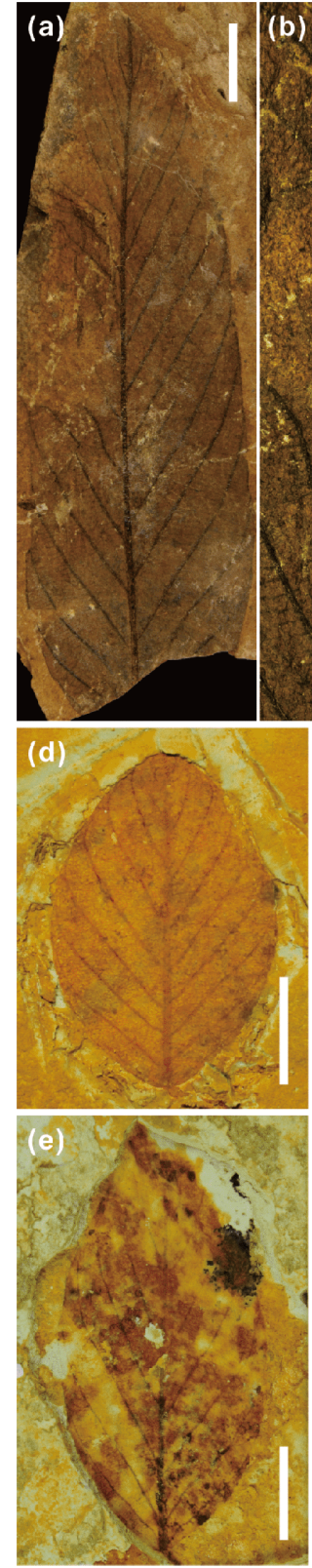
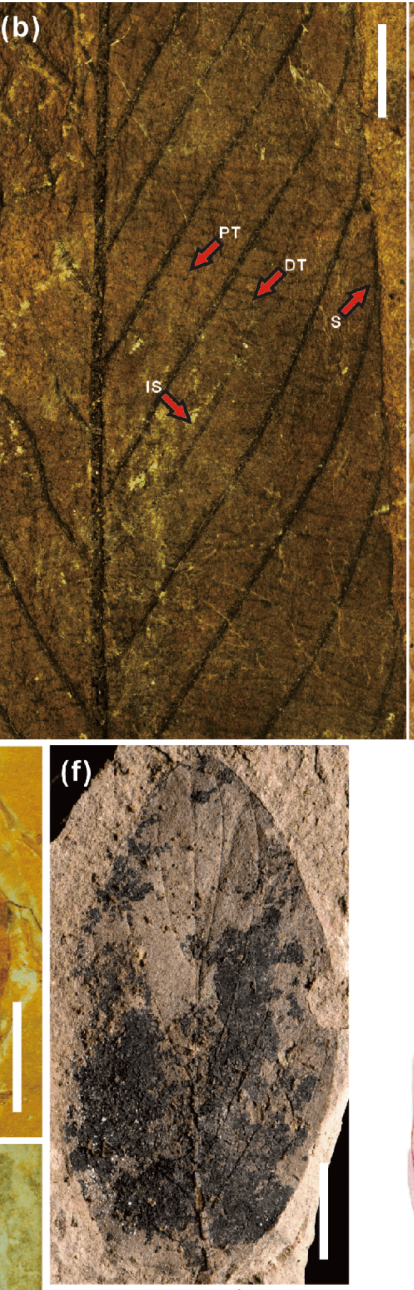

(g)

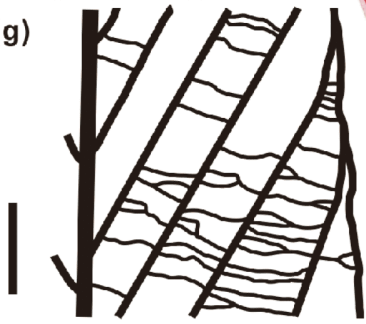

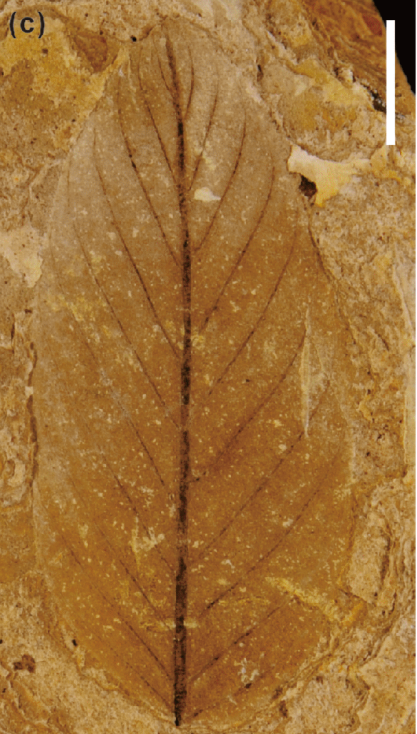

(h)

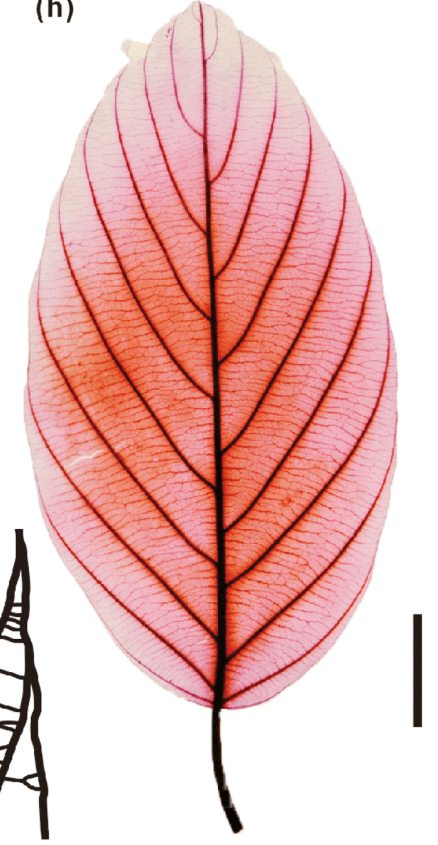

图 3 君容似勾儿茶叶(新种)(Berhamniphyllum junrongiae Z. K. Zhou, T.X. Wang et J. Huang sp. nov.)、多花似勾儿茶叶 (Berhamniphyllum miofloribundum (Hu et Chaney) J. Huang, T. Su et Z. K. Zhou comb. nov.)及长梗勾儿茶(Berchemia longipes) (现代)透明叶

（a) (c) 君容似勾儿茶叶(新种)：(a) MK3-1491号标本，比例尺=1cm；(b) MK3-1491细节，S(secondary vein)示汇入叶缘的二级脉; IS(inter secondary vein)示间二级脉; PT(percurrent tertiary vein)示贯穿的三级脉; DT(dichotomous tertiary vein)示二歧的三级脉, 比例尺 $=0.5 \mathrm{~cm} ;(\mathrm{c})$ MK3-2155 号标本, 比例尺 $=0.5 \mathrm{~cm}$; (d) (f) 多花似勾儿茶叶(新组合): (d) DMS-1713, 比例尺 $=0.5 \mathrm{~cm}$; (e) DMS-3872, 比例尺 $=0.5 \mathrm{~cm}$; (f) PB12519, 比例尺 $=0.5 \mathrm{~cm} ;(\mathrm{g}) \mathrm{MK} 3-1491$ 细节线描图, 比例尺 $=0.5 \mathrm{~cm}$; (h) 长梗勾儿茶 (Berchemia longipes) 透明叶, 比例尺 $=1 \mathrm{~cm}$

\section{4 讨论}

\section{1 鉴定与比较}

当前的化石标本叶缘全缘或微波状，二级脉之间
间距规则，弧曲向上逐步与其上的二级脉在叶缘处结 合并与叶缘融合形成真曲脉(eucamptodromous); 三级 脉密集相互平行，笔直贯穿于二级脉之间，这一叶相 特征组合在现代双子叶植物中也是较为独特的, 主要 
见于山茱英科(Cornaceae)的山茱英属(Cornus), 鼠李科 鼠李族的勾儿茶属(图3h)、小勾儿茶属和美洲分布的 Rhamnidium和Karwinskia. 但是, 山荣英属植物叶形多 为宽椭圆形或宽卵形, 二级脉较为稀疏, 具有六级高级 脉序, 这些特征与鼠李科植物有明显区别. 当前化石宏 观形态特征，特别是二级脉的特征与山荣英属的叶形 态特征相比有较大差距，而与鼠李族植物的若干属的 叶形态特征都非常相似，包括鼠李族中的小勾儿茶 属、勾儿茶属、Karwinskia属和Rhamnidium属等类群. 在现代植物中，小勾儿茶和勾儿茶在习性和花部特征 上都有区别，前者为直立灌木或小乔木，后者多为藤 本, 稀为直立灌木; 同时, 二属在叶基部形态也有区别, 前者叶基偏斜, 不对称, 后者叶基对称，叶基的特征在 化石上能够观察到, 当前化石叶基部对称, 因此排除了 小勾儿茶属的可能.

对于现代类群而言，虽然勾儿茶属和Karwinskia 属及Rhamnidium属的叶型和叶脉特征非常相似(图4), 但是从叶的排列方式上能够很容易地将勾儿茶属和后 两者分开. 勾儿茶属植物叶的排列方式为互生, 而后两 者为对生(图4), 勾儿茶属叶无腺点, 而后两者叶均有 腺点(Kubitzki, 2004). 在化石中, 叶的排列方式通常不 能被保存, 而以往研究经验表明, 在特定的环境和条件 下, 叶面的腺点有可能被保存下来, 但是在大多数情况 下, 腺点很难被保存, 因此腺点的有无, 很难作为区别 特征来广泛使用. Karwinskia属的四级脉发育明显，与 勾儿茶属和Rhamnidium属的四级脉序有区别，而勾儿 茶属和Rhamnidium属仅凭叶结构特征是不可能分开 的，Jones和Dilcher(1980)建立了一个化石形态属似勾 儿茶叶属(Berhamniphyllum)来囊括那些既像勾儿茶又
像Rhamnidium的叶化石，似勾儿茶叶属也有可能代表 的是一个亲缘关系和形态特征与勾儿茶属和Rhamnidium 都相近的一个灭绝类群. Jones和Dilcher的这种分 类学处理方式, 比较符合叶化石研究的实际, 而被后来 研究这类化石的研究者采纳和使用(Correa等，2010). 本文也采纳Jones和Dilcher的观点, 使用似勾儿茶叶属 来描述当前发现的化石.

芒康的标本有一个显著的特征即叶中部以上(叶 片纵轴 $50 \%$ 以上的部位)二级脉较为密集, 为4 8对, 占 二级脉总数的 $40 \%$ 以上(网络版附表, $\mathrm{http} / / /$ earthen.scichina.com), 而开远小龙潭和文山的标本叶中上部的二 级脉的对数为 $1 \sim 3$ 对, 仅占二级脉总数的 $20 \sim 33 \%$, 与芒 康的标本有明显区别, 因此将芒康的标本定为新种. 芒 康的标本仅有两块, 这两块标本的叶形也有区别, 一块 为矩圆形(图3c), 另一块标本为长卵形(图3a), 但是两 块标本的叶脉特征是非常相似的，包括叶中上部二级 脉密集这个重要鉴定特征都较为一致. 勾儿茶属植物 多为藤本植物，藤本植物的叶在不同的部位叶形会有 一定的变异, 芒康两块标本形态上的差异可以认为是 种内的差异.

小龙潭和文山的标本在形态特征上与中新世山东 山旺的多花藤非常相似, 因此都被定为多花藤 (Berchemia miofloribunda)(周浙昆，1985; 黄健，2017). 根据 Jones和Dilcher(1980)的分类学原则，本文将多花藤归 并到似勾儿茶叶属中, 即Berhamniphyllum miofloribundum.

\section{2 化石历史}

此处所讨论的化石历史包括了勾儿茶属和似勾儿
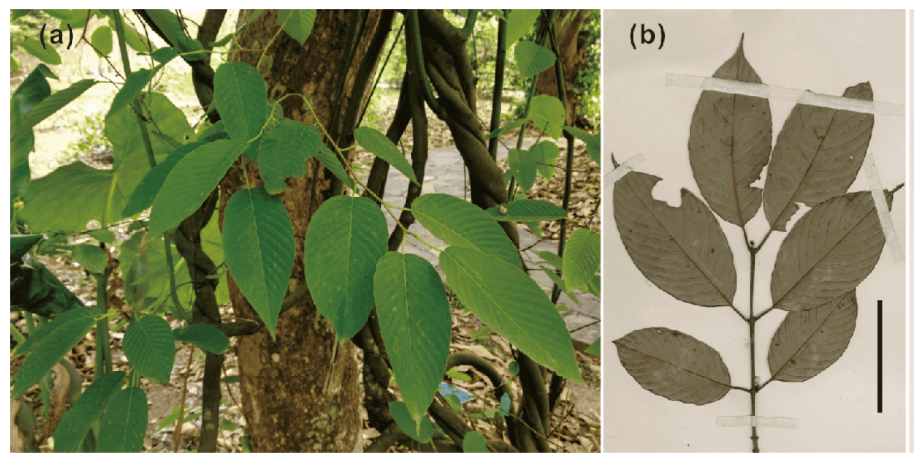

(c)

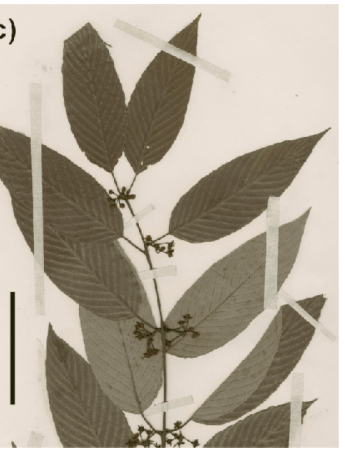

图 4 勾儿茶属、Karwinskia属及Rhamnidium属

(a) 长梗勾儿茶(Berchemia longipes), 图片摄自中国科学院西双版纳热带植物园, 示叶片互生; (b) Karwinskia calderonii, 比例尺 $=5 \mathrm{~cm}$, 图片来自 JSTOR, 示叶片对生; (c) Rhamnidium caloneurum, 比例尺 $=5 \mathrm{~cm}$, 图片来自JSTOR, 示叶片对生 


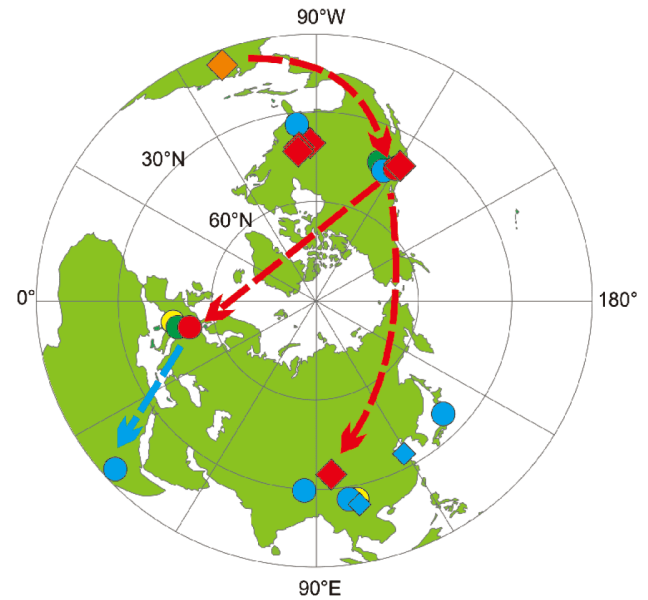

勾儿茶属化石

始新世

渐新世

中新世

似勾儿茶叶属化石

$\vee$ 晚白严世

- 始新世

中新世

图 5 鼠李族的勾儿茶类植物可能的传播路径 修改自Jia等(2015)

茶叶属(Berhamniphyllum). 如前所述, 勾儿茶属植物叶 脉特征明显且极为稳定，即二级脉弧向上与上一个相 邻的二级脉融合(真曲脉)并逐步与叶缘融为一体，三 级脉平行与二级脉近垂直相交(图3h)。这个特征使得 勾儿茶属的化石比较容易辨识，因而，大部分学者将 有这类叶脉特征的种类鉴定为勾儿茶属. 但是鼠李族 中的一个分布于南美和中美洲的小属Rhamnidium 的 叶形态特征和勾儿茶属植物的形态特征非常相似，仅 从叶形态特征看，两者在属级水平是分不开的. 更有 意思的是在哥伦比亚晚白严世和美国始新世也有类似 Rhamnidium 叶片特征的化石被报道(Jones和Dilcher, 1980；Correa等，2010)(图1；表2). Jones和Dilcher在对 Wilcox植物群(始新世)进行再研究时, 对Berry鉴定为 Rhamnus、Cornus的20多块标本进行了重新研究，认 为其中一些叶化石的标本与Rhamnidium 和Berchemia 都非常相似，而这两个属在叶形态特征上是完全不能 分开的, 于是建立了一个新形态属似勾儿茶叶属 $(B e r-$ hamniphyllum Jones and Dilcher), 用于描述那些既像 Rhamnidium, 也像勾儿茶属的叶化石，并认为这个形 态属代表了一个鼠李族的灭绝类群，而这个类群有可 能是Rhamnidium和勾儿茶属的共同祖先(Jones和Dilcher, 1980). 其后, Correa等(2010)在哥伦比亚晚白严世
的地层 $(68 \mathrm{Ma})$ 中也发现了似勾儿茶叶属的未定种 (Berhamniphyllum sp.)(表2). 美国古植物学家Manchester(2000)报道了产自美国俄勒冈Wheeler县的晚始新 世(39 38Ma)勾儿茶叶片化石, 这是在北美以勾儿茶属 命名的最早的化石记录. 美国蒙大拿西南部Beaverhead盆地中新统也有勾儿茶属的化石记录，这是北美 最晚的勾儿茶属的化石记录(Manchester, 2000).

欧洲最早可靠的勾儿茶属的化石记录产自于著名 的化石产地德国Messel中始新统(Collinson等，2012). 这是一个内果皮的化石, Collinson等人利用同步辐射 X光断层扫描技术(Synchrotron Radiation X-Ray Tomographic Microscopy, SRXTM)对其进行了详细的形态 解剖研究. 从始新世到上新世, 欧洲都有勾儿茶属叶化 石的记录，这些叶化石都是用勾儿茶属命名的(Givulescu, 1996; Bozukov, 2000; Teodoridis, 2007; Bozukov等, 2008; Kovar-Eder, 2016; Macaluso等, 2018)(表 1), 由于未见到欧洲及后面提及的日本的以Berchemia 命名叶化石标本, 本文不做归并. 化石记录表明上新世 以后, 勾儿茶属在欧洲灭绝.

非洲仅有两例勾儿茶属化石记录，分别是发现于 肯尼亚Rusinga岛的中新统Hiwegi组的种子化石以及 肯尼亚Fort Ternan中新统的外果皮化石, 均被定为 Berchemia psedodiscolor(Retallack, 1992; Collinson等, 2009)(表1).

勾儿茶属现在主要分布于东亚亚热带森林和东南 亚的热带森林, 在地质历史上, 勾儿茶及其近缘类群在 东亚也有丰富的化石记录(图1; 表1). 当前论文报道的 发现于西藏芒康的晚始新世叶化石，是亚洲最早的化 石记录(表1和2)，云南开远小龙潭、文山、临沧邦卖 和山东临朐山旺的中新世植物群均有勾儿茶属的记录 (Chaney和Hu, 1940; 中国新生代植物编写组, 1978; 陶 君容和陈明洪, 1983; 周浙昆, 1985; 郭双兴, 2011; 黄 健，2017)，本文中小龙潭、文山和山旺的勾儿茶属被 归并为似勾儿茶叶属，在上新世云南腾冲团田仍有勾 儿茶属的报道(吴靖宇, 2009). 在日本, 本州中新世开 始即有勾儿茶属的化石报告(Ishida, 1970; Ozaki, 1980, 1991; Yabe, 2008)(表1).

\section{3 现代分布格局的演变}

在讨论这个问题之前，有必要回顾一下鼠李科系 统发育关系的研究历史. 在较早的鼠李科分类系统中, 
勾儿茶属和束属植物因为都有核果, 而被放入到責族 中(Suessenguth, 1953), 中国植物志采纳了这一分类处 理. 其后, Richardson等(2000)根据分子系统学研究结 果将勾儿茶属从東族中分出, 移至鼠李族中, 鼠李族包 括了Auerodendron、Berchemia、Berchemiella、Condalia、Dallachya、Karwinskia、Krugiodendron、Reynosia、Rhamnidium、Rhamnella、Rhamnus、Sageretia和Scutia等13个属组成一个单系的支系. 勾儿茶属、 小勾儿茶属、Karwinskia和Rhamnidium等类群不仅叶 形态相似, 而且亲缘关系也非常接近, 为了便于讨论, 本文将以上4属称之为勾儿茶复合群(Berchemia complex), 并采纳Correa等(2010)的观点, 将似勾儿茶叶属 (Berhamniphyllum)看作勾儿茶复合群的共同祖先.

分子生物学和化石证据都表明, 鼠李科于晚白严 世早期起源于南美(Basinger和Dilcher, 1984; Hauenschild等, 2018). 鼠李族的勾儿茶复合群最早的化石记 录发现于哥伦比亚晚白严世最晚期, 可以认为勾儿茶 复合群起源时间不晚于晚白严世晚期, 起源地点极有 可能是南美洲的北部. 在始新世南北美洲之间通过一 些陆块和火山岛相连, 因此, 南北美洲的动植物区系 可以通过这些陆块和火山岛相交流(MacPhee和Iturralde-Vinent, 1995; Iturralde-Vinent和MacPhee, 1999). 勾儿茶复合群的有些种类极有可能也是通过这些陆块 扩散到北美的，这个扩散的路径和金虎尾科从南美向 北美的扩散路径一致(Davis等，2002；Zhou等，2006), 这一推测也得到了化石记录的印证.

在始新世北美和欧洲之间的区系交流主要是通过 大西洋陆桥来实现(Tiffney和Manchester, 2001), 对于 植物而言, 在中新世晚期之前北大西洋陆桥一直在发 挥着作用(Denk等, 2010). 而北美和东亚植物区系之间 的交流大多是通过白令陆桥来实现 (Donoghue和 Smith, 2004). 在早渐新世以前, 欧洲和亚洲之间被图 尔盖海峡隔开, 区系交流具有一定障碍(Tiffney和Manchester, 2001). 由于勾儿茶复合群在中始新世就已经 有了可靠的化石记录, 在本研究中, 始新世勾儿茶复 合群采取了两条不同的路径分别向欧洲和亚洲传播 (图5), 即通过北大西洋陆桥于中始新世就传播到欧洲, 东亚的勾儿茶复合群有可能是通过白令陆桥传播到亚 洲, 在日本以及中国东部和西南部都有勾儿茶的化石 记录, 是这种传播路径的佐证. 这个传播方式与椿榆 属(Cedrelospermum)、兔耳果属(Lagokarpos)从北美向
欧洲和亚洲的传播方式一致，椿榆的传播方式还得到 了形态学证据的支持(Jia等, 2015, 2019; Tang等, 2019).

根据化石记录, 勾儿茶复合群传播到青藏高原东 南缘的时间是晚始新世. 这个时期, 芒康的海拔比现 在低大约 $1000 \mathrm{~m}$, 当时的植被是青冈和桦木组成的常 绿落叶阔叶混交林(Su等, 2019b; Xu等, 2016). 古近纪 青藏高原有着比现在复杂的地形地貌, 中央存在着一 条海拔不超过 $2300 \mathrm{~m}$ 的东西向峡谷, 芒康位于这条峡 谷的最东端(Su等, 2019a). 峡谷中分布着西藏似沙巴 棕等植物, 指示峡谷内是一种温暖湿润的环境 $(\mathrm{Su}$ 等, 2019a). 在同时代, 中国的自然环境格局主要受行星风 系控制, 存在着一条横贯东西的干旱带(Sun和Wang, 2005; Guo等, 2008). 来自北美的勾儿茶复合群在东亚 可能最早定居于青藏高原的东南缘. 随着青藏高原进 一步隆升, 横跨东西的干旱带到中新世消失, 中国的 自然环境格局转变为中东部、东南部湿润而西北部干 旱(Sun和Wang, 2005; Guo等, 2008). 在中新世勾儿茶 复合群走出青藏高原, 传播到中国山东和云南以及日 本等地, 勾儿茶类植物进而逐步分化为今天的勾儿茶 属, 并在东亚得到较大的发展形成今天的分布格局.

\section{5 结论}

根据形态对比, 将发现于西藏芒康上始新统的标 本定为似勾儿茶叶属. 由于叶中上部二级脉比较密集, 不同于同属的所有类群, 于是建立一个新种: 君容似勾 儿茶叶(Berhamniphyllum junrongiae Z. K. Zhou, T.X. Wang et J. Huang sp. nov.). 君容似勾儿茶叶是勾儿茶 复合群在亚洲最早的化石记录, 从化石记录推测亚洲 的勾儿茶复合群是通过白令陆桥从北美扩散而至. 当 青藏高原隆升, 横贯东西的干旱带消失的时候, 扩散 至亚洲其他地区.

致谢感谢中国科学院西双版纳热带植物园和昆明植物 研究所的同事参与野外工作。感谢西藏芒康卡均藏族同 胞在野外工作中给予的帮助。感谢史恭乐博士帮助拍摄 云南开远小龙潭组勾儿茶的化石照片, Lutz Kunzmann教 授、王雨晴和Steven Manchester教授提供了部分欧洲、 日本和北美的文献. 在论文写作过程中, 与贾林波博士和 Steven Manchester教授进行过有益的讨论. 感谢中国科学 院西双版纳热带植物园中心实验室在摄影设备和技术上 
提供的支持。感谢两位若名审稿人在审稿过程中提出意 见和建议, 这些意见和建议对于拙稿的改进起到了重要 作用。

\section{参考文献}

郭双兴. 2011. 云南临沧晚中新世邦卖组植物群. 古生物学报, 50 : 353-408

黄健. 2017. 云南文山中新世植物群及古环境. 博士学位论文. 西双 版纳: 中国科学院西双版纳热带植物园. 1-320

陶君容, 陈明洪. 1983. 横断山南部-云南临沧地区新生代植物群. 中 国科学院青藏高原综合科学考察队. 横断山考察专集(一). 昆明: 云南人民出版社. 74-89

王伟铭. 1996. 云南开远小龙潭盆地晚第三纪狍粉植物群. 植物学报, 38: 743-748

吴靖宇. 2009. 云南腾冲上新世团田植物群及其古环境分析. 博士学 位论文. 兰州: 兰州大学. 1-122

吴征镒, 周浙昆, 孙航, 李德铢, 彭华. 2006. 种子植物分布区类型及 其起源和分化. 昆明: 云南科技出版社

中国新生代植物编写组. 1978. 中国植物化石. 第三册, 中国新生代 植物. 北京: 科学出版社

周浙昆. 1985. 云南开远小龙潭中新世植物群. 博士学位论文. 南京: 中国科学院南京地质古生物研究所

Ai K K, Shi G L, Zhang K X, Ji J L, Song B W, Shen T Y, Guo S X. 2019. The uppermost Oligocene Kailas flora from southern Tibetan Plateau and its implications for the uplift history of the southern Lhasa terrane. Palaeogeogr Palaeoclimatol Palaeoecol, 515: 143151

Basinger J F, Dilcher D L. 1984. Ancient bisexual flowers. Science, 224: $511-513$

Becker H F. 1969. Fossil plants of the Tertiary Beaverhead Basins in souothwestern Montana. Palaeontogr Abt B, 127: 1-142

Berry E W. 1916a. The lower Eocene floras of southeastern North America. US Geol Surv Prof Pap, 91: 1-149

Berry E W. 1916b. The physical conditions indicated by the flora of the Calvert formation. US Geol Surv Prof Pap, 98: 61-73

Bozukov V. 2000. Miocene macroflora of the Satovcha Graben (Western Rhodopes). Phytol Balcan, 5: 15-30

Bozukov V, Palamarev E, Petkova A. 2008. The fossil macroflora of the Vulche Pole Molasse formation (SE Bulgaria). Phytol Balcan, 14: 173-184

Büchler W. 1990. Eine fossile Flora aus dem oberen Oligozän von Ebnat-Kappel. Bot Helv, 100: 133-166

Chaney R W, Hu H H. 1940. A Miocene Flora from Shantung Province, China. Washington: Publication of Carnegie Institute. 1-507
Chen Y, Schirarend C. 2007. Rhamnaceae. In: Wu Z Y, Raven P H, Hong D Y, eds. Flora of China. Beijing: Science Press. St. Louis: Missouri Botanical Garden Press. 12: 115-168

Collinson M E, Andrews P, Bamford M K. 2009. Taphonomy of the early miocene flora, Hiwegi formation, Rusinga Island, Kenya. J Human Evol, 57: 149-162

Collinson M E, Manchester S R, Wilde V. 2012. Fossil fruits and seeds of the Middle Eocene Messel biota, Germany. Abh Senckenb Ges Naturforsch, 570: 1-251

Correa E, Jaramillo C, Manchester S, Gutierrez M. 2010. A fruit and leaves of Rhamnaceous affinities from the late Cretaceous (Maastrichtian) of Colombia. Am J Bot, 97: 71-79

Davis C C, Bell C D, Mathews S, Donoghue M J. 2002. Laurasian migration explains Gondwanan disjunctions: Evidence from Malpighiaceae. Proc Natl Acad Sci USA, 99: 6833-6837

Denk T, Grímsson F, Zetter R. 2010. Episodic migration of oaks to Iceland: Evidence for a North Atlantic "land bridge" in the latest Miocene. Am J Bot, 97: 276-287

Deng T, Wang X, Wu F, Wang Y, Li Q, Wang S, Hou S. 2019. Review: Implications of vertebrate fossils for paleo-elevations of the Tibetan Plateau. Glob Planet Change, 174: 58-69

Dilcher D L, Lott T A. 2005. A middle Eocene fossil plant assemblage (Powers Clay Pit) from western Tennessee. Bull Florida Museum Nat Hist, 45: 1-43

Ding L, Spicer R A, Yang J, Xu Q, Cai F, Li S, Lai Q, Wang H, Spicer T E V, Yue Y, Shukla A, Srivastava G, Khan M A, Bera S, Mehrotra R. 2017. Quantifying the rise of the Himalaya orogen and implications for the South Asian monsoon. Geology, 45: 215-218

Dong W, Qi G. 2013. Hominoid-producing localities and biostratigraphy in Yunnan. In: Wang X M, Flynn L J, Fortelius M, eds. Fossil Mammals of Asia-Neogene Biostratigraphy and Chronology. New York: Colombia University Press. 293-313

Donoghue M J, Smith S A. 2004. Patterns in the assembly of temperate forest around the Northern Hemisphere. Phil Trans R Soc Lond B, 359: $1633-1644$

Ellis B, Daly D C, Hickey L J, Johnson K R, Mitchell J D, Wilf P, Wing S L. 2009. Manual of Leaf Architecture. Ithaca: Cornell University Press

Flora of North America Editorial Committee, eds. 1993+. Flora of North America North of Mexico. 19+ vols. New York: Oxford University Press

Givulescu R. 1996. Flora Oligocena Superioara din Bazinul Petrosani. Casa Cartii de Stiinta, Cluj-Napoca, 1-177

Guo Z T, Sun B, Zhang Z S, Peng S Z, Xiao G Q, Ge J Y, Hao Q Z, Qiao Y S, Liang M Y, Liu J F, Yin Q Z, Wei J J. 2008. A major reorganization of Asian climate by the early Miocene. Clim Past, 4: 
$153-174$

Hauenschild F, Favre A, Michalak I, Muellner-Riehl A N. 2018. The influence of the Gondwanan breakup on the biogeographic history of the ziziphoids (Rhamnaceae). J Biogeogr, 45: 2669-2677

Hantke R. 1954. Die fossile Flora der obermiozänen OehningerFundstelle Schrotzburg (Schienerberg, Süd-Baden). Doctoral Dissertation. Zürich: ETH Zürich

Heer O. 1855-1859. Flora Tertiaria Helveticae. Die Tertiäre flora der Schweiz. Winterthur: J. Wurster and Compagnie

Ishida S. 1970. The Noroshi flora of Noto Peninsula, Central Japan. Memoirs of the Faculty of Science Kyoto University, Series of Geology and Mineralogy, 37: 1-112

Iturralde-Vinent M A, MacPhee R D E. 1999. Paleogeography of the Caribbean region: Implications for Cenozoic biogeography. Bull Am Mus Nat Hist, 238: 1-95

Jia L B, Manchester S R, Su T, Xing Y W, Chen W Y, Huang Y J, Zhou Z K. 2015. First occurrence of Cedrelospermum (Ulmaceae) in Asia and its biogeographic implications. J Plant Res, 128: 747-761

Jia L B, Su T, Huang Y J, Wu F X, Deng T, Zhou Z K. 2019. First fossil record of Cedrelospermum (Ulmaceae) from the Qinghai-Tibetan Plateau: Implications for morphological evolution and biogeography. J Syt Evol, 57: 94-104

Jiang H, Su T, Wong W O, Wu F, Huang J, Shi G. 2019. Oligocene Koelreuteria (Sapindaceae) from the Lunpola Basin in central Tibet and its implication for early diversification of the genus. J Asian Earth Sci, 175: 99-108

Jones J H, Dilcher D L. 1980. Investigations of angiosperms from the Eocene of North America: Rhamnus marginatus (Rhamnaceae) reexamined. Am J Bot, 67: 959-967

Jung W. 1968. Pflanzenreste aus dem Jungtertiär Nieder-und Oberbayerns und deren lokalstratigraphische Bedeutung. Ber Naturwiss Ver Langshut, 25: 43-71

Köecke V, Uhl D. 2015. The leaf assemblage from the Early-Middle Miocene locality Sulzigtobel near Werthenstein (Canton Lucerne, Switzerland). Phytol Balcan, 21: 99-109

Kovar-Eder J. 2016. Early Oligocene plant diversity along the Upper Rhine Graben: The fossil flora of Rauenberg, Germany. Acta Palaeobot, 56: 329-440

Kubitzki K. 2004. The Families and Genera of Vascular Plants. Vol. 6. Flowering Plants-Dicotyledons: Celastrales, Oxalidales, Rosales, Cornales, Ericales. Berlin: Springer

Lazarević Z, Milivojević J, Bogićević K, Nenadić D. 2013. Early Miocene flora from the Valjevo-Mionica Basin (Western Serbia). N J Geol Pal A, 267: 297-307

Lebreton-Anberrée J, Li S, Li S F, Spicer R A, Zhang S T, Su T, Deng C, Zhou Z K. 2016. Lake geochemistry reveals marked environ- mental change in Southwest China during the Mid Miocene Climatic Optimum. Chin Sci Bull, 61: 897-910

de León P V, Cevallos-Ferriz S R, Silva-Pineda A. 1998. Leaves of Karwinskia axamilpense sp.nov. (Rhamnaceae) from Oligocene sediments, near Tepexi de Rodríguez, Puebla, Mexico. Can J Bot, 76: $410-419$

Li S F, Mao L M, Spicer R A, Lebreton-Anberrée J, Su T, Sun M, Zhou Z K. 2015. Late Miocene vegetation dynamics under monsoonal climate in southwestern China. Palaeogeogr Palaeoclimatol Palaeoecol, 425: 14-40

Li S H, Deng C L, Dong W, Sun L, Liu S Z, Qin H F, Yin J Y, Ji X P, Zhu R X. 2015. Magnetostratigraphy of the Xiaolongtan Formation bearing Lufengpithecus keiyuanensis in Yunnan, southwestern China: Constraint on the initiation time of the southern segment of the Xianshuihe-Xiaojiang fault. Tectonophysics, 655: 213-226

Liu J, Su T, Spicer R A, Tang H, Deng W Y D, Wu F X, Srivastava G, Spicer T, Van Do T, Deng T, Zhou Z K. 2019. Biotic interchange through lowlands of Tibetan Plateau suture zones during Paleogene. Palaeogeogr Palaeoclimatol Palaeoecol, 524: 33-40

Macaluso L, Martinetto E, Vigna B, Bertini A, Cilia A, Teodoridis V, Kvaček Z. 2018. Palaeofloral and stratigraphic context of a new fossil forest from the Pliocene of NW Italy. Rev Palaeobot Palynol, 248: $15-33$

MacPhee R D E, Iturralde-Vinent M A. 1995. Origin of the Great Antillean land mammals, 1: New Tertiary fossils from Cuba and Puerto Rico. Am Mus Novitates, 3141: 1-31

Manchester S R. 2000. Late Eocene fossil plants of the John Day Formation, Wheeler County, Oregon. Oregon Geol, 62: 51-63

Myers J A, Kester P R, Retallack G J. 2002. Paleobotanical record of Eocene-Oligocene climate and vegetational change near Eugene, Oregon. Oregon Dep Geol Min Ind Spec Pap, 36: 145-154

Myers N, Mittermeier R A, Mittermeier C G, da Fonseca G A B, Kent J. 2000. Biodiversity hotspots for conservation priorities. Nature, 403 : 853-858

Ozaki K. 1980. Late Miocene Tatsumitoge flora of Tottori Prefecture, Southwest Honshu, Japan (III). Sci Rep Yokohama Natl Univ, 27: $19-45$

Ozaki K. 1991. Late Miocene and Pliocene Floras in Central Honshu, Japan. Bulletin of Kanagawa Prefectural Museum Natural Science Special Issue. Yokohama: Kanagawa Prefectural Museum. 1-244

Palgrave K C. 2015. Palgrave's Trees of Southern Africa. 3rd ed. Cape Town: Struik Publishers

Prasad M, Dwivedi H D. 2007. Systematic study of the leaf impressions from the Churia Formation of Koilabas area, Nepal and their significance. Palaeobotanist, 56: 139-154 
Retallack G J. 1992. Middle Miocene fossil plants from Fort Ternan (Kenya) and evolution of African grasslands. Paleobiology, 18: $383-400$

Richardson J E, Fay M F, Cronk Q C B, Chase M W. 2000. A revision of the tribal classification of rhamnaceae. Kew Bull, 55: 311-340

Sakala J. 2000. Flora and vegetation of the roof of the main lignite seam in the Bilina Mine (Most Basin, Lower Miocene). Acta Mus Nat Pragae Ser B Hist Nat, 56: 49-84

Singh S K, Prasad M. 2007. Late Tertiary leaf flora of mahuadanr valley, Jharkhand. J Palaeontol Soc India, 52: 175-194

Smiley C J, Gray J, Huggins L M. 1975. Preservation of Miocene fossils in unoxidized lake deposits, Clarkia, Idaho. J Paleontol, 49: 833-844

Spicer R A. 2017. Tibet, the Himalaya, Asian monsoons and biodiversity-In what ways are they related? Plant Divers, 39: 233-244

Spitzelberger V G. 1989. Die Miozänfundstelle Goldern bei Landshut (Niederbayern). Geol Bavarica, 94: 371-407

Su T, Wilf P, Xu H, Zhou Z K. 2014. Miocene leaves of Elaeagnus (Elaeagnaceae) from the Qinghai-Tibet Plateau, its modern center of diversity and endemism. Am J Bot, 101: 1350-1361

Su T, Li S F, Tang H, Huang Y J, Li S H, Deng C L, Zhou Z K. 2018. Hemitrapa Miki (Lythraceae) from the earliest Oligocene of southeastern Qinghai-Tibetan Plateau and its phytogeographic implications. Rev Palaeobot Palynol, 257: 57-63

Su T, Farnsworth A, Spicer R A, Huang J, Wu F X, Liu J, Li S F, Xing Y W, Huang Y J, Deng W Y D, Tang H, Xu C L, Zhao F, Srivastava G, Valdes P J, Deng T, Zhou Z K. 2019a. No high Tibetan Plateau until the Neogene. Sci Adv, 5: eaav2189

Su T, Spicer R A, Li S H, Xu H, Huang J, Sherlock S, Huang Y J, Li S F, Wang L, Jia L B, Deng W Y D, Liu J, Deng C L, Zhang S T, Valdes P J, Zhou Z K. 2019b. Uplift, climate and biotic changes at the Eocene-Oligocene transition in south-eastern Tibet. Natl Sci Rev, 6: 495-504

Suessenguth K. 1953. Rhamnaceae. In: Engler A, Prantl K, eds. Die natiirlichen Pflanzenfamilien. 2nd ed. Berlin: Dunker et Humboldt Sun X J, Wang P X. 2005. How old is the Asian monsoon system?-
Palaeobotanical records from China. Palaeogeogr Palaeoclimatol Palaeoecol, 222: 181-222

Tang H, Liu J, Wu F, Spicer T, Spicer R A, Deng W, Xu C, Zhao F, Huang J, Li S, Su T, Zhou Z. 2019. Extinct genus Lagokarpos reveals a biogeographic connection between Tibet and other regions in the Northern Hemisphere during the Paleogene. J Syt Evol, 18: jse. 12505

Taylor T N, Taylor E L, Krings M. 2008. Paleobotany: The Biology and Evolution of Fossil Plants. 2nd ed. New York: Academic Press. 1230

Teodoridis V. 2007. Revision of Potamogeton fossils from the Most Basin and their palaeoecological significance (Early Miocene, Czech Republic). Bull Geosci, 82: 409-418

Tiffney B H, Manchester S R. 2001. The use of geological and paleontological evidence in evaluating plant phylogeographic hypotheses in the Northern hemisphere tertiary. Int J Plant Sci, 162: S3-S17

Wu F X, Miao D S, Chang M M, Shi G L, Wang N. 2017. Fossil climbing perch and associated plant megafossils indicate a warm and wet central Tibet during the late Oligocene. Sci Rep, 7: 878

Xu C L, Su T, Huang J, Huang Y J, Li S F, Zhao Y S, Zhou Z K. 2019. Occurrence of Christella (Thelypteridaceae) in Southwest China and its indications of the paleoenvironment of the Qinghai-Tibetan Plateau and adjacent areas. J Syt Evol, 57: 169-179

Xu H, Su T, Zhang S T, Deng M, Zhou Z K. 2016. The first fossil record of ring-cupped oak (Quercus L. subgenus Cyclobalanopsis (Oersted) Schneider) in Tibet and its paleoenvironmental implications. Palaeogeogr Palaeoclimatol Palaeoecol, 442: 61-71

Xu H, Su T, Zhou Z K. 2019. Leaf and infructescence fossils of Alnus (Betulaceae) from the late Eocene of the southeastern QinghaiTibetan Plateau. J Syt Evol, 57: 105-113

Yabe A. 2008. Early Miocene terrestrial climate inferred from plant megafossil assemblages of the Joban and Soma areas, Northeast Honshu, Japan. Bull Geol Surv Jpn, 59: 397-413

Zhou Z K, Yang X F, Yang Q S. 2006. Land bridge and long-distance dispersal-Old views, new evidence. Chin Sci Bull, 51: 10301038 